\title{
The Role of Meta-Motivational States in Sexual Risk-Taking Intentions During Condom Negotiation
}

\author{
Shayna Skakoon-Sparling \\ University of Guelph \& University of Windsor
}

\author{
Kenneth M. Cramer \\ University of Windsor
}

\begin{abstract}
The current study applied reversal theory to better understand the motivational factors that influence sexual health decision-making during condom negotiation among young men and women. Participants $(N=440)$ read a vignette describing a romantic sexual encounter with a hypothetical new partner and answered embedded questions related to their attitudes and behavioral intentions. Participants who were experiencing a stronger goal-oriented (telic) state showed a more risk adverse response pattern: they perceived unprotected sex as posing a greater risk and were more likely to refuse to have unprotected sex in the hypothetical encounter. Participants who were experiencing a stronger conformist state also responded in a more risk adverse pattern: they showed a greater interest in using a condom, were more likely to initiate condom negotiation, and were less willing to agree to have unprotected sex in the hypothetical scenario. The findings of the current study also suggest that the rules domain may function based on established societal norms rather than situational norms, since the social pressure of the hypothetical scenario could have encouraged more conformist individuals to adhere to the hypothetical partner's wishes to engage in unprotected sex. The findings of current study demonstrate the utility of reversal theory and suggest that incorporating the influence of meta-motivational states into sexual risk-taking models will allow for a deeper understanding of why individuals choose to engage in behaviors that put their sexual health at risk which, in turn, will inform future interventions to encourage protective behaviors.
\end{abstract}

Keywords: Reversal Theory; sexual risk-taking; condom negotiation; motivated reasoning

The health threat posed by sexually transmitted infections (STIs) remains a concern among young people, thus it is important that we strive to better understand the decisionmaking route that leads to unprotected sex, which is the most common method of STI transmission (Centers for Disease Control and Prevention, 2016; Gahagan et al., 2013; Kerry, Nightingale, \& Oakeshott, 2016; Milhausen et al., 2013; Public Health Agency of Canada, 2015). Sexual health decision-making is an important component in the negotiation of condom use and can be affected by both contextual and internal factors (e.g., sexual arousal: Skakoon-Sparling, Cramer, \& Shuper, 2016) and Reversal Theory offers a unique lens through which we may better understand condom negotiation and sexual health decision-making. Thus, the aim of the current study was to explore the role of metamotivational states in condom negotiation among young men and women.

Shayna Skakoon-Sparling, Dept. Family Relations and Applied Nutrition, University of Guelph; Kenneth M. Cramer, Department of Psychology, University of Windsor.

Correspondence concerning this article should be addressed to Shayna Skakoon-Sparling at sparling.s@gmail.com
Reversal theory describes personality and motivation as states that are fluid and subject to change from moment to moment (Apter, 2007). Reversal theory examines the dynamic shifts (or reversals) between meta-motivational states to better understand individuals' inconsistent behavior. These shifts can impact an individual's motivations and subsequent behavior. Within the framework of sexual risktaking, there are two meta-motivational domains believed to be associated with risk-taking: the means-end domain and the rules domain.

The means-end domain contains both the telic state (associated with future thinking and goal-oriented behavior) and the paratelic state (characterized by a focus on pleasure and a desire to enjoy the present moment). Paratelic state dominance has been associated with risky sexual behavior (Gerkovich, 2001; Lafreniere, Cramer, \& Out, as cited in Lafreniere, Menna, \& Cramer, 2013), as well as elevated sexual arousal levels (Skakoon-Sparling \& Cramer, 2015), which is also associated with increased sexual risktaking intentions and decreased self-control (e.g., SkakoonSparling \& Cramer, 2016; Skakoon-Sparling, Cramer, \& Shuper, 2016).

The rules domain contains both the agreeable and rulefollowing conformist state and the stubborn and rule defying negativistic state (also known as rebelliousness) (Apter 2007; 
Gerkovich, 2001). In a sexual encounter, the dominance of the negativistic state could allow an individual to rationalize unprotected sex because it might feel exciting to knowingly engage in this risky behavior or to flout social norms around safe sex (O'Connell, Schwartz, Gerkovich, Bott, \& Shiffman, 2004). Very little work has examined the link between negativism and sexual risk-taking; however, there is some evidence that individuals who are dominant in negativism are more likely to report engaging in risky sexual behavior (Lafreniere et al., 2013).

It is critical that more work be done to explore these metamotivational states and their influence on human behavior and decision-making. For instance, it is unknown whether individuals experiencing a strong conformist state will conform to broad social norms or to specific situational norms. The difference between these two contexts is important, as this can have very different implications for risk-taking behavior. For instance, an individual who perceives strong social norms in favor of condom use may be better able to insist on condom use with a resistant partner when experiencing a strong conformist state. In contrast, a conformist individual who adheres to situational norms would be unmotivated to insist on condom use if she perceives her partner as uninterested in condom use. It is clear that the valuable insights from reversal theory must be added to the current body of literature related to sexual risk-taking.

The Current Study. The current study investigated the impact of telic/paratelic and conformist/negativist motivational state balance on the process of condom negotiation among both heterosexual couples and men who have sex with men using a hypothetical sexual scenario. We hypothesized that participants experiencing a stronger paratelic state or a stronger negativistic state would select less assertive condom insistence strategies. We also hypothesized that individuals experiencing stronger telic or conformist states would show lower risk taking intentions.

\section{Methods}

\section{Participants}

Participants $(N=440)$ were recruited online: 307 participants (101 women who have sex with men (WSM), 104 men who have sex with women (MSW), and 102 men who have sex with men (MSM)) using Amazon's Mechanical Turk (MTurk) system and 133 participants (76 WSM, 53 MSW, and 4 MSM) using the Participant Pool system at a southern Ontario university in Canada.

In order to be eligible, participants had to identify as being between 18 and 25 years of age and as a cis-gendered (where sex assigned at birth matches currently identified gender) man or woman. Eligible participants also had to indicate that they had engaged in consensual vaginal or anal sex at least once ever and that they were not currently involved in a long-term monogamous relationship (participants must either be single or in a casual dating relationship).

The mean age of MTurk participants was 23 years $(S D=$ 1.80 ), and $85 \%$ of this sample self-identified as single. Fortytwo percent of the sample indicated that they were having no sexual relations, and $45 \%$ of the sample indicated that they were, but not with any exclusive partner(s). The vast majority of this sample indicated their current country of residence was the United States (99\%). In terms of ethnicity, this sample primarily identified as white $(72 \%)$. Thirty-four percent of MTurk respondents indicated that over the past three months they tended to use condoms "sometimes" or less frequently; $66 \%$ indicated that they used condoms "often" or more frequently.

The mean age of Pool participants was 20 years $(S D=$ 1.55 ), and $64 \%$ of this sample identified as single. Thirtyseven percent of the total sample indicated that they were having no sexual relations and $29 \%$ indicated that they were, but not with any exclusive partner(s). This entire sample indicated that their current country of residence was Canada $(100 \%)$. In terms of ethnicity, this sample primarily identified as white $(82 \%)$. Forty-eight percent of Pool respondents indicated that over the past three months they tended to use condoms "sometimes" or less frequently; 53\% indicated that they use condoms "often" or more frequently.

\section{Materials}

Hypothetical Scenario. Participants read a vignette describing a hypothetical romantic encounter with a new sexual partner. The vignettes were largely identical across all participants, with a few small changes to tailor the scenario to participants' gender and sexual orientation (e.g., for WSM and MSM the hypothetical partner is a man named Chris; for MSW it is a woman named Alia). In the presented scenario, unprotected sex with the hypothetical partner should objectively be considered a risky sexual behavior, since participants had no information about the hypothetical partner's sexual health history (e.g., number of past partners, recency of STI testing, most recent STI test result, etc.) (Comer \& Nemeroff, 2000). Throughout the scenario, items were presented that probed participants' attitudes and behavioral intentions. At two different points in the scenario participants are also asked to select a condom insistence strategy from a list of options. At Time 1, participants select a strategy for suggesting condom use to their partner; at Time 2, participants select a strategy to insist on condom use, after the hypothetical partner has indicated $\mathrm{s} / \mathrm{he}$ is resistant to condom use. After completing the scenario, participants rated how easy it was to picture themselves in the scenario on a scale from 1 ('could not see self in scenario at all') to 10 ('could very easily see self in scenario') and generally found it easy to project themselves into the presented scenario $(M=8.59, S D=1.73)$. 
Table 1

Linear Regression Results

\begin{tabular}{lcccccc}
\hline & \multicolumn{2}{c}{$\begin{array}{c}\text { Own } \\
\text { Interest in } \\
\text { Condom Use }\end{array}$} & \multicolumn{2}{c}{$\begin{array}{c}\text { Partner } \\
\text { Interest in } \\
\text { Condom Use }\end{array}$} & \multicolumn{2}{c}{$\begin{array}{c}\text { Risk } \\
\text { Perception }\end{array}$} \\
\hline Variables & $b(S E)$ & $\beta$ & $b(S E)$ & $\beta$ & $b(S E)$ & $\beta$ \\
\hline Telic/Paratelic & $-1.71(1.37)$ & -.076 & $2.98(1.40)$ & $.131^{*}$ & $.28(.11)$ & $.165^{* *}$ \\
Confomity/Negativism & $2.52(1.28)$ & $-.121^{*}$ & $.38(1.31)$ & .018 & $-.15(.10)$ & -.093 \\
Adjusted $R^{2}$ & 0.028 & & 0.01 & & 0.051 & \\
F Statistic & $7.26^{* *}$ & & $3.23^{*}$ & & $12.70^{* * *}$ & \\
\hline
\end{tabular}

$* p<.05 * * p<.01 * * * p<.001$

Table 2

Linear Regression Results (continued)

\begin{tabular}{lcccccc}
\hline & \multicolumn{2}{c}{$\begin{array}{c}\text { Likelihood of } \\
\text { Suggesting } \\
\text { Condom Use }\end{array}$} & \multicolumn{2}{c}{$\begin{array}{c}\text { Willingness to } \\
\text { to Have } \\
\text { Unprotected Sex }\end{array}$} & $\begin{array}{c}\text { Willingness to } \\
\text { to Leave } \\
\text { Without Sex }\end{array}$ \\
\hline Variables & $b(S E)$ & $\beta$ & $b(S E)$ & $\beta$ & $b(S E)$ & $\beta$ \\
\hline Telic/Paratelic & $1.17(1.33)$ & .053 & $-.13(.14)$ & -.053 & $.35(.15)$ & $.146^{*}$ \\
Confomity/Negativism & $-3.97(1.25)$ & $-.193^{* *}$ & $.32(.14)$ & $.143^{*}$ & $-.15(.14)$ & -.065 \\
Adjusted $R^{2}$ & 0.049 & & 0.029 & & 0.034 & \\
F Statistic & $12.27^{* * *}$ & & $7.48^{* *}$ & & $8.57^{* * *}$ & \\
\hline
\end{tabular}

$* p<.05 * * p<.01 * * * p<.001$

Motivational State Assessment. Participants completed the Telic/Paratelic State Inventory-Modified (T/PSI$\mathrm{M})$, which is based on the scale by O'Connell and Calhoun (2001) (for the full scale see Kerr, 1999). T/PSI-M items are scored on a dichotomous 9-point scale (e.g., $1=$ "feeling playful", 9 = "feeling serious"); a lower score on this scale indicates a stronger Paratelic state balance. The same version of the T/PSI-M was used by Skakoon-Sparling and Cramer (2014) and demonstrated good reliability (alpha $=.87$ ). The T/PSI-M also showed good reliability in the current study $($ alpha $=.86)$. Items from the T/PSI-M were intermixed with items from the Negativist-Conformist State inventory (N/CSI), a 10 item scale, which is also scored on a 9-point dichotomous scale (e.g., $1=$ "following the rules" vs. $9=$ "making my own rules"; 1 = "doing what is expected of me" vs. 9 = "doing whatever I want"); a lower score on this scale indicates a stronger Conformist state balance. During pilot testing, 124 undergraduate participants (68 women, 56 men) completed the newly developed N/CSI scale: it was found to have acceptable internal reliability (alpha $=.76)$. In the current study, the N/CSI scale also achieved an alpha of .76.

\section{Procedure}

Participants who clicked through the invitation link viewed a brief introduction screen and consent form. Participants then read a vignette depicting a romantic encounter with a hypothetical new sexual/romantic partner and responded to items embedded in the scenario. After completing the vignette, all participants completed the metamotivational state inventories and provided demographic information that included their sexual history and practices, as well as measures of other sexual risk related factors, such as a depression inventory, a measure of poly drug use, and a measures of past experience of intimate partner violence.

\section{Results}

\section{Interest in Condom Use}

Participants were asked to estimate how much they would want to use a condom if sex were to occur during the hypothetical sexual encounter $(0=$ 'not at all', $100=$ 'very much'), as well as how much they thought their hypothetical partner would want to use a condom on this occasion.

A multiple linear regression was undertaken for 439 participants to examine variance in participants' stated interest in condom use (see Table 1 for results). A stronger Negativistic state balance was associated with a decreased stated interest in condom use. Telic/Paratelic state balance did not emerge as a significant predictor.

A multiple linear regression was undertaken for 439 participants to examine variance in participants' perception of their partner's interest in condom use (see Table 1 for re- 
Table 3

\begin{tabular}{lcccc} 
Summary of Condom Insistence Strategies - Telic/Paratelic State \\
\hline Response & \multicolumn{2}{c}{ Time 1} & \multicolumn{2}{c}{ Time 2} \\
\hline & Conformist & Negativist & Conformist & Negativist \\
None - Would Have Unprotected Sex & $5(4 \%)$ & $35(12 \%)$ & $7(6 \%)$ & $51(17 \%)$ \\
Withholding Sex & $40(32 \%)$ & $66(22 \%)$ & $44(35 \%)$ & $83(28 \%)$ \\
Direct Request & $33(26 \%)$ & $74(25 \%)$ & $23(18 \%)$ & $46(16 \%)$ \\
Seduction & $33(26 \%)$ & $81(28 \%)$ & $24(19 \%)$ & $58(20 \%)$ \\
Relationship Conceptualizing & $5(4 \%)$ & $11(4 \%)$ & $5(4 \%)$ & $8(3 \%)$ \\
Risk (STI) Information & $5(4 \%)$ & $10(3 \%)$ & $8(6 \%)$ & $13(4 \%)$ \\
Deception & $6(4 \%)$ & $17(6 \%)$ & $15(12 \%)$ & $29(10 \%)$ \\
\hline
\end{tabular}

sults). A stronger Telic state balance was associated with an increased perception that the hypothetical partner would be interested in condom use. Conformist/Negativist state balance did not emerge as a significant predictor.

\section{Risk Perception}

Risk Perception was assessed using respondents' rating of how risky they felt it would be to have unprotected sex with the hypothetical partner ( 1 = "not at all risky", $10=$ "extremely risky"). A multiple linear regression was undertaken for 439 participants to examine variance in participants' perception of the risk associated with unprotected sex with the hypothetical partner (see Table 1 for results). A stronger Telic state balance was associated with an increased risk perception. Conformist/Negativist state balance did not emerge as a significant predictor.

\section{Likelihood of Suggesting Condom Use}

Likelihood of suggesting condom use was assessed using participant responses to an item asking the likelihood that they would bring up using a condom with the hypothetical partner during the sexual encounter $(0=$ "extremely unlikely", 100 = "extremely likely"). A multiple linear regression was undertaken for 439 participants to examine variance in participants' stated likelihood of suggesting condom use (see Table 2 for results). A stronger Conformist state balance was associated with an increased stated likelihood of suggesting condom use. Telic/Paratelic state balance did not emerge as a significant predictor.

\section{Telic/Paratelic State Balance and Condom Negotiation Strategy}

A Telic-Paratelic state balance (TPB) score was calculated by averaging together the responses to all items. TPB score did not differ by gender/sexuality identity $(p>.05)$. In order to examine how participants' condom negotiation strategies differed based on TPB, TPB was dichotomized: scores of 4.99 and lower were categorized as High Paratelic $(n=295)$ and scores of 5.01 and higher were categorized as High Telic $(n=128)$. Respondents whose average score was exactly 5 were excluded $(n=17)$, since they appeared to be experiencing the exact midpoint between the Telic and Paratelic states.

A Pearson's $\chi^{2}$ indicated no significant effect of TPB at Time 1: $\chi^{2}(6, N=421)=9.18, p=.164, \phi=.148$. A Pearson's $\chi^{2}$ indicated a marginally significant effect of TPB at Time 2: $\chi^{2}(7, N=422)=13.16, p=.068, \phi=.177$. (Telic/Paratelic State Balance accounted for $3.13 \%$ of the variance in condom negotiation strategy at Time 2.) Specifically, it was found that participants who scored more strongly Paratelic were somewhat less likely to select any condom negotiation strategy (i.e., were more willing to have unprotected sex). See summary Table 3 for detail.

\section{Conformity/Negativism State Balance and Condom Ne- gotiation Strategy}

The C/NSI scale items were averaged to obtain an overall Conformity Negativism Balance (CNB) score. CNB score did not differ by gender/sexuality identity $(p>.05)$. In order to examine how participants' condom negotiation strategies differed based on CNB, CNB was dichotomized: scores of 4.99 and lower were categorized as High Conformity $(n=188)$ and scores of 5.01 and higher were categorized as High Negativism $(n=228)$. Respondents whose average score was exactly 5 were excluded $(n=24)$, since they appeared to be experiencing the exact midpoint between the Conformist and Negativistic states.

A Pearson's $\chi^{2}$ indicated a significant effect of CNB at Time 1: $\chi^{2}(6, N=414)=17.55, p<.01, \phi=.206$. (Conformist/Negativistic State Balance accounts for $4.24 \%$ of the variance in condom negotiation strategy at Time 1.) Specifically, it was found that participants who scored more strongly Negativistic were significantly less likely to select any condom negotiation strategy (i.e., were more willing to have unprotected sex).

A Pearson's $\chi^{2}$ indicated a significant effect of CNB at Time 2: $\chi^{2}(7, N=415)=15.57, p<.05, \phi=.193$. Conformist/Negativistic State Balance accounts for $3.73 \%$ of the variance in condom negotiation strategy at Time 2 . 
Table 4

\begin{tabular}{lcccc} 
Summary of Condom Insistence Strategies - Conformity/Negativism State \\
\hline Response & \multicolumn{2}{c}{ Time 1} & \multicolumn{2}{c}{ Time 2} \\
\hline & Conformist & Negativist & Conformist & Negativist \\
None - Would Have Unprotected Sex & $8(4 \%)$ & $34(15 \%)$ & $13(7 \%)$ & $43(19 \%)$ \\
Withholding Sex & $56(30 \%)$ & $49(22 \%)$ & $65(35 \%)$ & $57(25 \%)$ \\
Direct Request & $45(24 \%)$ & $64(28 \%)$ & $33(18 \%)$ & $36(16 \%)$ \\
Seduction & $55(29 \%)$ & $53(23 \%)$ & $40(21 \%)$ & $44(19 \%)$ \\
Relationship Conceptualizing & $7(4 \%)$ & $9(4 \%)$ & $5(3 \%)$ & $9(4 \%)$ \\
Risk (STI) Information & $8(4 \%)$ & $6(3 \%)$ & $10(5 \%)$ & $12(5 \%)$ \\
Deception & $8(4 \%)$ & $12(5 \%)$ & $17(9 \%)$ & $22(10 \%)$ \\
\hline
\end{tabular}

Specifically, it was found that participants who scored more strongly Negativistic were significantly less likely to select any condom negotiation strategy. Additionally, participants who scored more strongly Conformist were more likely to select the Withholding Sex strategy. See summary Table 4 for more detail.

\section{Willingness to Engage in Unprotected Sex}

After the first time participants were asked to select a condom influence strategy, they were informed that their hypothetical partner was reluctant to use a condom (they were also told that there might not be a condom available), and the hypothetical partner attempted to convince the participant to have unprotected sex. Participants then indicated how likely they would be to agree to have unprotected sex using a 10point Likert scale ( 1 = "extremely unlikely", 10 = "extremely likely"). A multiple linear regression was undertaken for 439 participants to examine variance in participants' willingness to have unprotected sex with the hypothetical partner (see Table 2 for results). A stronger Negativist state balance was associated with an increased willingness to have unprotected sex. Telic/Paratelic state balance did not emerge as a significant predictor.

\section{Willingness to Refuse Unprotected Sex}

Participants were also asked to indicate how likely they would be to just leave without having sex with the hypothetical partner. A multiple linear regression was undertaken for 439 participants to examine variance in participants' willingness to refuse unprotected sex with the hypothetical partner (see Table 2 for results). A stronger Telic state balance was associated with an increased willingness to refuse unprotected sex. Conformist/Negativist state balance did not emerge as a significant predictor.

\section{Exploratory Analyses ${ }^{1}$}

Association between Meta-Motivational States. A moderate negative correlation was found between Telic/Paratelic state balance and Conformist/Negativist state balance: $r p(439)=-.640, p<.001$. Stronger Telic state balance was associated with stronger Conformist state balance. This finding seems logical, as someone who is strongly goal oriented may see conforming to social rules as a good way to set and achieve goals.

Risk Factors Associated with Meta-Motivational States. In order to explore the potential association between typical risk factors associated with increased sexual risk-taking (e.g., depression, early sexual debut, condom use frequency, poly drug use, and intimate partner violence) variables related to these risk factors were entered into multiple linear regression analyses in order to determine which would emerge as the best predictors of Telic/Paratelic and Conformist/Negativist state balance respectively.

A multiple linear regression was undertaken for 418 participants to examine variance in participants' Telic/Paratelic state balance using the Stepwise method. A significant model $F(2,417)=5.41, p<.01)$ accounted for $2.5 \%$ of the outcome variance (Adj. $R^{2}=.021$ ). Two predictors were entered into the final model: poly drug use $(\beta=-.132, p<.01)$ and past experience of sexual battery by a partner $(\beta=$ $.108, p>.01$ ). Thus, poly drug use (using more than 1 type of drug in the past three months) was significantly associated with a stronger Paratelic state.

A multiple linear regression was undertaken for 418 participants to examine variance in participants' Conformist/Negativist state balance using the Stepwise method. A significant model $F(3,417)=8.48, p<.01$ accounted for $5.8 \%$ of the outcome variance (Adj. $R^{2}=.051$ ). Three predictors were entered into the final mode: poly drug use $(\beta=.207, p<.01)$, past experience of physical battery by a partner $(\beta=-.122, p=.012)$ and depression score $(\beta=.114, p=.018)$. Thus, poly drug use (using more than 1 type of drug in the past three months) was significantly associated with a stronger Negativist state, higher scores on a depression inventory (the 10-item version of The Center for Epidemiologic Studies Depression Scale; Andersen, Malmgren, Carter, \& Patrick, 1994) was also marginally significantly associated with a stronger Negativistic State.

\footnotetext{
${ }^{1}$ For all exploratory analyses alpha was set at .01.
} 
Past experience of physical battery at the hands of a partner was marginally significantly associated with a stronger Conformist state.

Correlation between Recent Condom Use Estimates. In the demographics section, two similar measures of recent condom use were deployed. One (condom use frequency) asked participants to estimate how frequently they tended to use condoms during consensual sexual encounters over the past three months. The other measure asked participants to note the number of consensual sexual encounters they had participated in over the past three months, as well as the number of these encounters where they did not use a condom. Using this count data, we determined the proportion of sexual encounters where unprotected sex occurred. We were curious about how these two measures would relate with one another and conducted a 2-tailed bivariate Pearson's correlation. We observed a moderate correlation between reported condom use frequency in the past three months and the proportion of recent sexual encounters where no condom was used: $r(345)=-.52, p<.001$. This suggests that these two methods for recording respondents' sexual health practices may still be providing unique information; since the correlation was only moderate, it may be that respondents' actual behavior, as measured by the count data, did not always correspond perfectly with their own perceptions of their general condom use habits, as measured by the condom use frequency item.

\section{Discussion}

The current study examined the relevance of two metamotivational state domains, the means-end domain and the rules domain, to sexual health decision-making in the condom negotiation process. Participants were presented with a vignette where they engaged in simulated condom negotiation with a hypothetical new partner and subsequently completed meta-motivational state inventories.

It was found that participants' meta-motivational state balance did have some degree of influence over their responses to the items embedded within the hypothetical scenario vignette. As predicted, participants who were experiencing a stronger Negativistic state balance showed a more risk seeking response pattern. Additionally, participants who were experiencing a stronger Telic state balance showed a more risk adverse response pattern.

Participants who experienced different meta-motivational states also selected different condom insistence strategies. Participants who experienced a stronger Paratelic state at Time 2 were more likely to select no strategy and agree to unprotected sex. This result is expected, based on the findings of Skakoon-Sparling and Cramer (2014); as individuals experiencing a stronger Paratelic state are more interested in enjoying the moment and thus may be also less interested in engaging in condom negotiation with a partner who is resis- tant to condom use. It is no surprise that a stronger Paratelic state was also associated with a decreased perception that a partner would be interested in condom use, were less likely to perceive that unprotected sex with the hypothetical partner would be risky, and were less willing to refuse to have unprotected sex and leave the hypothetical encounter. These results are expected, given the focus on immediate pleasure rather than hypothetical risk that is typically associated with the Paratelic state.

It may also be that some aspects of the scenario invoked a protective frame, which allowed some participants to shift to a stronger Paratelic state, where they felt they could afford to be less concerned about the riskiness of their behavioral intentions. A protective frame is an element that can allow an individual to feel safe reversing to the Paratelic state from the Telic state (Apter, 2014). A protective frame can exist as physical space (e.g., an amusement park, a movie theatre, or a bedroom), it can manifest from objects (e.g., a condom, a helmet, or a map), and it can arise from more abstract experiences and sensations (e.g., practice/rehearsal, confidence, or trust in another person). It may be that participants inferred lower risk based on the characteristics of the hypothetical partner (individuals tend to assume a partner is STI-free if they do not disclose otherwise: Downing-Matibag \& Geisinger, 2009; see also Skakoon-Sparling \& Cramer, 2015), which may have invoked a protective frame. The hypothetical nature of the scenario itself may have generated a protective frame, since a hypothetical encounter would have a lower emotional impact compared with a real-life situation (Collett \& Childs, 2011).

Participants who were experiencing a strong Negativistic state were more likely to agree to unprotected sex (no strategy) and participants experiencing a stronger Conformist state were more likely to select withholding sex as a strategy. This pattern of response fits well with the characteristics associated with these two states: the Conformist state is associated with rule following and adhering to prescriptive norms (Gerkovich, 2001), thus it should not be surprising that individuals experiencing this state would select a more assertive strategy to insist on condom use, despite their hypothetical partner's resistance to condom use. Strongly insisting on condom use conforms to established societal norms about practising safe sex, particularly with a new partner (rather than situational norms in this context-since participants could have conformed to the wishes of the hypothetical partner instead). Similarly, it is not surprising that participants experiencing a stronger Negativistic state were more willing to flout these same social norms. Indeed, this fits with the pattern of responses seen for the other scenario items. Participants experiencing a stronger Negativistic state seemed to be more willing to flout other social norms related to condom use and safer sexual practises - demonstrated by their lower interest both using or suggesting condom use and 
their greater willingness to have unprotected sex in the hypothetical scenario. This finding not only confirms the association between the Negativistic state and increased sexual risk-taking, but also suggests that the norms that impact the rules domain are societal rather than situational. Further research is needed to confirm this proposed association; however, the impact of social norms is a commonly considered factor in many models related to health decision-making. For instance, the Information-Motivation-behavioral Skills (IMB) Model (Fisher \& Fisher, 1992; 1993) is used to help understand HIV risk behavior and serves as a guide for the development of interventions to change this behavior. This model incorporates perceived social norms into the element of social motivation, which is part of the critical Motivation portion of this model. The IMB model proposes that an individual's perceptions about social support and social norms for protective behaviors will influence their motivation to engage in such behaviors. Reversal theory and the findings of the current study add a critical element to this concept-an individual's state balance within the rules domain may influence the way perceived social norms impact motivation and subsequent protective or risk-taking behavior.

\section{Limitations}

This study is not without limitations. For instance, although a great deal of detail was included in the vignettes used in the current study, it is impossible to fully capture all of the subtleties that would be present in a real-life situation (Hughes \& Huby, 2004). Thus, as mentioned above, the scenarios participants read may not have evoked as strong an emotional response (Collett \& Childs, 2011). However, the results of this study are not without value, since the sexual risk-taking intentions do tend to be predictive of actual behavior (e.g., Sheeran, Abraham, \& Orbell, 1999; Sheeran \& Orbell, 1998; Turchik \& Gidycz, 2012). Despite these limitations, the current study contributes important elements to both the reversal theory literature on the means-end and the rules domains as well as to our understanding of how reversal theory can inform our understanding of sexual decisionmaking and condom negotiation.

\section{Conclusion and Implications}

The results of the current study indicate that metamotivational state balance can have an impact on sexual risktaking intentions and condom negotiation. Participants experiencing either a more goal-oriented or a more social norm conforming meta-motivational state tended to place more value on condom use. In contrast, participants experiencing either a more pleasure oriented state or a more rebellious state tended to show greater sexual risk-taking intentions. This highlights the contribution reversal theory can offer to better understanding how motivations for engaging in sexual risk-taking behavior can impact adherence to sexual health goals and, subsequently, condom negotiation. These findings also indicate that situational cues may allow individuals to generate or perceive a protective frame, which can serve to justify sexual risk-taking. Future work is needed to better understand the factors that contribute to the generation of a protective frame in sexual situations, as well as the types of cues that could be added via interventions to undermine the protective frame to some degree in order to encourage safer sexual behavior without overly inhibiting individuals' enjoyment of a sexual encounter. The current findings also improve our understanding of the rules domain, because it seems that the norms individuals may be either conforming with or rebelling against tend to be social norms rather than situational norms. Further study is needed to better explore this relationship.

\section{References}

Andersen, E. M., Malmgren, J. A., Carter, W. B., \& Patrick, D. L. (1994). Screening for depression in well older adults: Evaluation of a short form of the CES-D (Center for Epidemiologic Studies Depression Scale). American Journal of Preventive Medicine, 10, 77-84.

Apter, M. J. (2007). Reversal theory: The dynamics of motivation, emotion, and personality. Oxford: Oneworld.

Apter, M. J. (2014). Towards a theory of things: Reversal theory and design. Journal of Motivation, Emotion, and Personality, 2, 3-11.

Centers for Disease Control and Prevention. (2016). Sexually Transmitted Disease Surveillance 2015. Atlanta: U.S. Department of Health and Human Services.

Collett, J. L., \& Childs, E. (2011). Minding the gap: Meaning, affect, and the potential shortcomings of vignettes. Social Science Research, 40, 513-522.

Comer, L. K., \& Nemeroff, C. J. (2000). Blurring emotional safety with physical safety in AIDS and STD risk estimations. Journal of Applied Social Psychology, 30, 24672490.

Downing-Matibag, T. M., \& Geisinger, B. (2009). Hooking up and sexual risk taking among college students: A health belief model perspective. Qualitative Health Research, 19, 1196-1209.

Fisher, W. A., \& Fisher, J. D. (1993). A general social psychological model for changing AIDS risk behavior. In: J.B. Pryor \& G.D. Reeder (Eds.) The Social Psychology of HIV Infection. Hillsdale, New Jersey: Erlbaum.

Fisher, W. A., \& Fisher, J. D. (1992). Understanding and promoting AIDS preventive behaviour: A conceptual model and educational tools. The Canadian Journal of Human Sexuality, 1, 99-106.

Gahagan, J., \& The Gathering of Spirits Collaborative. (2013). The National Consensus Statement on Women, Trans People and Girls and HIV Research. Halifax, NS: 
Dalhousie University, Gender and Health Promotion Studies Unit.

Gerkovich, M. M. (2001). Risk-Taking. In M. J. Apter (Editor), Motivational Styles in Everyday Life (p. 215-228). Washington, DC: American Psychological Association.

Hughes, R., \& Huby, M. (2004). The construction and interpretation of vignettes in social research. Social Work and Social Sciences Review, 11, 36-51.

Kerr, J. H. (1999) Appendix B, Telic/Paratelic State Instrument. In, Experiencing Sport: Reversal Theory, Chichester, UK: Wiley.

Kerry, S. R., Nightingale, C. M., \& Oakeshott, P. (2016). Which sexually active female students get themselves tested for Chlamydia trachomatis? A cohort study. International Journal of STDs and AIDS, 27, 586-590.

Lafreniere, K. D., Menna, R., \& Cramer, K. M. (2013). Rebelliousness, effortful control, and risky behaviour: Metamotivational and temporal predictors of risk-taking in older adolescents. Journal of Motivation, Emotion, and Personality, 1, 17-26.

Milhausen, R. R., McKay, A., Graham, C. A., Crosby, R. A., Yarber, W. L., \& Sanders, S. A. (2013). Prevalence and predictors of condom use among a national sample of Canadian university students. The Canadian Journal of Human Sexuality, 22(3), 142-151.

O'Connell, K. A., \& Calhoun, J. E. (2001). The telic/paratelic state instrument (T/PSI): Validating a reversal theory measure. Journal of Personality and Individual Differences, 30, 193-204.
Public Health Agency of Canada. (2015). Report on Sexually Transmitted Infections in Canada: 2012. Centre for Communicable Diseases and Infection Control, Infectious Disease Prevention and Control Branch, Public Health Agency of Canada.

Sheeran, P., Abraham, C., \& Orbell, S. (1999). Psychosocial correlates of heterosexual condom use: A meta-analysis. Psychological Bulletin, 125, 90-132.

Sheeran, P., \& Orbell, S. (1998). Do intentions predict condom use? Meta-analysis and examination of six moderator variables. Journal of Social Psychology, 37, 231-250.

Skakoon-Sparling, S., \& Cramer, K. M. (2016). The impact of sexual arousal on elements of sexual decision making: sexual self-restraint, motivational state, and self-control. The Canadian Journal of Human Sexuality, 25, 119-125.

Skakoon-Sparling, S., Cramer, K. M., \& Shuper, P. A. (2016). The impact of sexual arousal on male and female sexual risk-taking and decision-making. Archives of Sexual Behavior, 45, 33-42.

Skakoon-Sparling, S., \& Cramer, K. (2014) Paratelic/telic state, sexual arousal, and sexual risk-taking in university students. Journal of Motivation, Emotion, and Personality, 2, 62-67.

Turchik, J. A., \& Gidycz, C. A. (2012). Exploring the intention-behavior relationship in the prediction of sexual risk behaviors: Can it be strengthened? Journal of Sex Research, 49, 50-60. 